dabei dem Bestehen eines bilateralen Kapitalschutzvertrages zukomme; auch hierzu sind allenfalls Fragmente einer Problemlösung aufzufinden (z. B. 71)

Alles in allem: Das Gebiet der außenwirtschaftlichen Subventionen harrt weiterhin einer erschöpfenden Durchdringung.

Ludwig Gramlich

\title{
Maurice Barbier
}

\section{Le Conflit du Sahara Occidental}

Editions l'Harmattan, Paris, 1982, 419 S., FF 133.-

\section{Tony Hodges}

\section{Western Sahara - The Roots of a Desert War}

Lawrence Hill \& Comp., Westport, Connecticut (USA) 1983 und Croom Helm Ltd., Beckenham (GB), 1983, 388 S., $\mathfrak{E} 16.95$

Nur wenige deutschsprachige Untersuchungen widmeten sich bisher dem seit 1975 andauernden Konflikt um die ehemalige spanische Kolonie Westsahara. Wissenschaftliches Interesse fanden die Ansprüche Marokkos auf das rohstoff reiche Nachbarland hierzulande lediglich nach der Veröffentlichung eines Gutachtens des Internationalen Gerichtshofs von 1975, in dem historische, das Selbstbestimmungsrecht des sahrauischen Volkes verdrängende Ansprüche des Königreichs verneint wurden. Darüber hinaus wurde verschiedentlich der Frage nachgegangen, in - welchem Zusammenhang die gescheiterten Versuche der OAU zur Konfliktbeilegung mit der Krise dieser Staatenorganisation stehen.

Wer mehr über Geschichte und Hintergründe des Konflikts und die Anstrengungen internationaler Organisationen zu seiner Beilegung erfahren will, muß auf ausländische Darstellungen zurückgreifen. Unter ihnen ragen zwei exzellente, umfassende Arbeiten besonders hervor.

Maurice Barbier, der Politikwissenschaften an der Universität Nancy-II lehrt, gräbt nach den Wurzeln des Konflikts. Aufschluß über Handlungsmotive der Konfliktbeteiligten verspricht sich Barbier aus einer detaillierten Rekonstruktion der vorkolonialen Geschichte der Region und ihres Kolonisierungsprozesses. Nachdem eingangs die ursprünglichen Sozialstrukturen und Eigenarten der sahrauischen Stämme sowie die ökonomischen Ressourcen der westlichen Sahara beschrieben werden, geht Barbier bis in das 14. Jahrhundert zurück, um nach Beispielen europäischer Einmischung zu forschen. Barbier konnte eine Fülle von Spuren sichern und den Lesern internationale Verträge präsentieren, die sich zunächst mit dem Schutz gestrandeter europäischer Seefahrer befaßten. Dieses und die nachfolgenden Kapitel über die Anfänge der Kolonisierung Nordwestafrikas durch Spanien und Frankreich, die vertraglichen Aufteilungen der "Interes- 
sensphären" und die Politik der Kolonialmacht Spanien in der "Spanischen Sahara" gegenüber den Nachbarländern, verdeutlichen die Auswirkungen der Kolonisierung auf das Leben der Sahrauis und die internationalen Beziehungen zwischen Algerien, Marokko, Mauretanien und Spanien. Im 2. Teil seiner Untersuchung zeichnet Barbier das Bemühen der UNO, die eine Mission in die Westsahara und die Anrainerstaaten sandte, um eine Streitbeilegung nach, bringt er das Gutachten des "Internationalen Gerichtshofs" in Erinnerung, analysiert er die Schachzüge der marokkanischen Diplomatie und beschreibt er die vergeblichen Vermittlungsversuche seit Beginn des bewaff neten Widerstands der Sahrauis gegen die marokkanische Okkupation. Der 3. Teil behandelt verschiedene Aspekte nach dem Kriegsbeginn 1975: die Staatsgründung der Demokratischen Arabischen Republik Sahara und ihre diplomatischen Erfolge, die Organisationsform der Befreiungsbewegung $F$. Polisario und ihre militärischen Aktionen, die Verwaltung in den Flüchtlingslagern, die Internationalisierung des Konflikts sowie die Aktivitäten der UN und der OAU. Eine umfangreiche, gegliederte und kommentierte Bibliographie beendet das Buch.

Barbier kommt zu dem Schluß, daß es sich um eine in der Regionalgeschichte tief verwurzelte Konfrontation der Nationalismen handle. Anderen Faktoren wie innenpolitische Kämpfe in Marokko, wirtschaftliche und militärstrategische Interessen Frankreichs und der USA geht er nur am Rande nach. "Neo-Kolonialismus" oder "Imperialismus" sind für ihn keine zentralen Kategorien zur Erklärung des Sahara-Kriegs. In offensiver Auseinandersetzung mit pro-marokkanischen Quellen tritt Barbier aber für das Recht des sahrauischen Volks auf Selbstbestimmung und das Recht auf einen unabhängigen Staat ein.

Beeindruckend ist an der Arbeit Barbiers, daß zwischen der nüchternen Abhandlung des wissenschaftlichen Themas immer wieder die katastrophalen Folgen der uneinsichtigen marokkanischen Politik verdeutlicht werden: Hungernde Menschen aufgrund der ruinierten Nationalökonomien zunächst in Mauretanien und dann in Marokko und unsagbare Härten für das exilierte sahrauische Volk.

Während sich Barbier auf völkerrechtliche Aspekte konzentriert, kann Hodges beanspruchen, das bisher umfassendste Bild des Westsahara-Konflikts gezeichnet zu haben. Hodges hat auf seiner vielbeachteten Vorarbeit, dem 1982 in den USA erschienenen 500 Seiten starken "Historical Dictionary of Western Sahara" aufbauen können. Trotz der gewaltigen Zeitspanne - von den Jahren der arabischen Einwanderung in Nordwestafrika bis 1983 - und des weitgesteckten thematischen Rahmens bleibt die faktenreiche Arbeit konzentriert und entwickelt klare Analysen. Mit Barbier stimmt er zwar darin überein, daß es ein die Westsahara einschließendes "Groß-Marokko" niemals gab und betont, daß die Sahrauis über Jahrhunderte ihre spezifische gesellschaftliche Organisation und eine besondere, sich von der der Nachbarregionen unterscheidende Kultur herausgebildet haben. Dennoch hält er diese Aspekte für zweitrangig bei der Begründung einer nachkolonialen Eigenständigkeit. Wesentlich ist für Hodges die Herausbildung des modernen sahrauischen Nationalismus, der ein Produkt der wirtschaftlichen und sozialen Veränderungen seit den letzten Jahren der spanischen Kolonialherrschaft sei. Er 
untersucht die wirtschaftliche Entwicklung der "Spanischen Sahara" seit den 60er Jahren: Phosphat-Boom, der Aufschwung in anderen Wirtschaftsbereichen, Ausbau der Verwaltung und Verstädterung der Bevölkerung. Hodges beschreibt den politischen Emanzipationsprozeß der Sahrauis, in dem die Frauen eine hervorragende aktive Rolle spielten. Damit tritt Hodges der marokkanischen These entgegen, daß die Sahrauis Objekt diplomatischer Winkelzüge und keine eigenständig handelnde politische Kraft seien. Darstellungen der Streitbeilegungsversuche der OAU und den UN fehlen ebensowenig wie die wichtigen Beschreibungen der marokkanischen und der spanischen Diplomatie. Als besonders verläßlich dürfen seine Berichte über das Kriegsgeschehen und das Leben in den Lagern gelten, da Hodges bei seinen vier Jahre dauernden Reisen hinter allen Fronten auch Beobachtungen ohne offizielle Begleitung machen konnte. Das Buch schließt mit einem Kapitel über die Aktivitäten der Weltmächte im SaharaKrieg, in dem zwangsläufig die US-Politik den größten Raum einnimmt. Hodges, der mehrfach vom amerikanischen Kongreß als Sachverständiger zum Sahara-Konflikt herangezogen wurde, gibt eine umfassende Darstellung der Sahara-Politik der USA unter Ford, Carter und Reagan, insbesondere der militärischen Unterstützung, und dokumentiert auch ausführlich die Position der - durchaus zahlreichen - Gegner dieser Marokko unterstützenden Politik im US-Parlament.

Hodges gibt keine expliziten Prognosen für die weitere Entwicklung des Konflikts, unternimmt aber eine Charakterisierung der am Krieg beteiligten Parteien und ihrer Bündnispartner. Bei der F. Polisario, deren Führer er großteils persönlich kennt und mit deren interner Entwicklung er besser vertraut sein dürfte als die meisten anderen Beobachter, betont er die Kontinuität und den Zusammenhalt der politischen Spitze, die behutsame Verbindung moderner Bildungs- und Verwaltungssysteme mit alten sahrauischen Gesellschaftsstrukturen in den Flüchtlingslagern und vor allem die hohe militärische Effizienz des Befreiungskampfs. Algerien als wichtigster Bündnispartner der Sahrauis bescheinigt er eine konsequente und stetige Saharapolitik seit 1975. Das marokkanische Regime stehe ökonomisch und politisch auf tönernen Füßen.

Beide Bände verdienen es, auch im deutschsprachigen Raum beachtet zu werden. 